PREPARED FOR THE U.S. DEPARTMENT OF ENERGY, UNDER CONTRACT DE-AC02-76CH03073

PPPL-3873

PPPL-3873

UC-70

Theoretical and Numerical Properties of a Gyrokinetic Plasma:

Issues Related to Transport Time Scale Simulation

by

W.W. Lee

September 2003

M

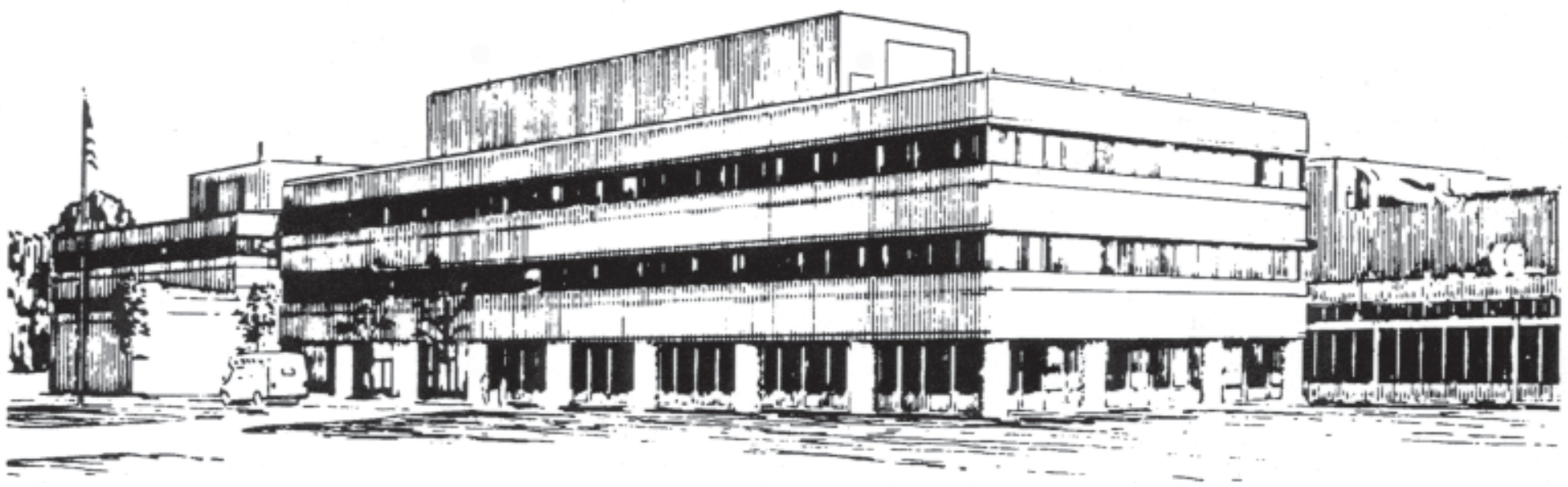

PRINCETON PLASMA PHYSICS LABORATORY PRINCETON UNIVERSITY, PRINCETON, NEW JERSEY 


\section{PPPL Reports Disclaimer}

This report was prepared as an account of work sponsored by an agency of the United States Government. Neither the United States Government nor any agency thereof, nor any of their employees, makes any warranty, express or implied, or assumes any legal liability or responsibility for the accuracy, completeness, or usefulness of any information, apparatus, product, or process disclosed, or represents that its use would not infringe privately owned rights. Reference herein to any specific commercial product, process, or service by trade name, trademark, manufacturer, or otherwise, does not necessarily constitute or imply its endorsement, recommendation, or favoring by the United States Government or any agency thereof. The views and opinions of authors expressed herein do not necessarily state or reflect those of the United States Government or any agency thereof.

\section{Availability}

This report is posted on the U.S. Department of Energy's Princeton Plasma Physics Laboratory Publications and Reports web site in Fiscal Year 2003. The home page for PPPL Reports and Publications is: http://www.pppl.gov/pub_report/

DOE and DOE Contractors can obtain copies of this report from:

U.S. Department of Energy

Office of Scientific and Technical Information

DOE Technical Information Services (DTIS)

P.O. Box 62

Oak Ridge, TN 37831

Telephone: (865) 576-8401

Fax: (865) 576-5728

Email: reports@adonis.osti.gov

This report is available to the general public from:

National Technical Information Service

U.S. Department of Commerce

5285 Port Royal Road

Springfield, VA 22161

Telephone: $1-800-553-6847$ or

(703) $605-6000$

Fax: (703) 321-8547

Internet: http://www.ntis.gov/ordering.htm 


\title{
Theoretical and Numerical Properties of a Gyrokinetic Plasma: Issues Related to Transport Time Scale Simulation
}

\author{
W. W. Lee \\ Princeton Plasma Physics Laboratory \\ Princeton, NJ 08543
}

\begin{abstract}
Particle simulation has played an important role for the recent investigations on turbulence transport in magnetically confined plasmas. In this paper, theoretical and numerical properties of a gyrokinetic plasma as well as its relationship with magnetohydrodynamics (MHD) are discussed with the ultimate aim of simulating microturbulence in transport time scale using massively parallel computers.
\end{abstract}

Key words: Gyrokinetics, Particle Simulation

PACS: 52.30.Gz, 52.65.y

\section{Introduction}

The study of theoretical and thermodynamic properties of simulation plasmas has a long history, since they are intrinsically related to the numerical schemes used for particle simulation. The effects on the collisionless simulation due to finite-size particles [1] and the discovery of convective cells in magnetized plasmas [2] are a few of the examples in the past. In this paper, we will describe the thermodynamic and numerical properties of a gyrokinetic plasma in terms of fluctuation-dissipation theorem, entropy production, energy conservation and the finite Larmor radius (FLR) effects related to turbulence simulations. Some of these issues have already been addressed before. For example, the drastic noise reduction in an electrostatic gyrokinetic simulation plasmas [3,4] has been explained by the change in the linear dielectric function [5]. The decade old puzzle concerning the noisy electron response in a finite $\beta$ gyrokinetic plasma [6-8] has recently been resolved [9] which leads to the formulation of the perturbative split-weight simulation scheme [9]. In view of the recent work on the relationship of compressional and shear Alfvén waves between MHD 


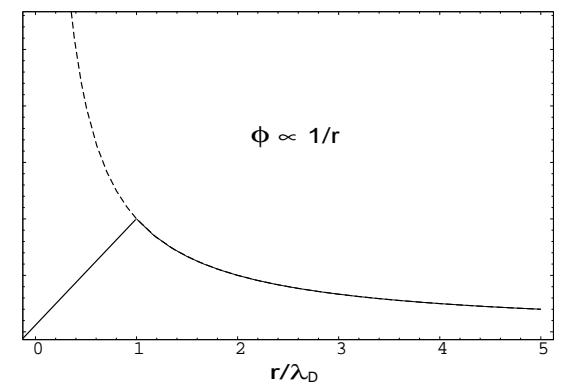

Fig. 1. Modification of the Debye shielding effect due to finite-size particles

and gyrokinetic descriptions [10], we will review these properties and explore the possibility of simulating kinetic-MHD physics via particle codes such as the transport time scale simulation of microturbulence.

\section{Fluctuation properties of a simulation plasma}

The methodology of particle-in-cell (PIC) simulation was first presented in the 1968 APS meeting by Dawson et al. [11] and Birdsall et al. [12] as a way to eliminate enhanced fluctuations (noise) due to close encounters of the simulation particles, i.e., the discrete particle effects. As we mentioned earlier, the use of finite-size particles has been theoretically verified by Langdon and Birdsall [1] via the fluctuation-dissipation theorem, i.e.,

$$
V \frac{|\mathbf{E}(\mathbf{k})|^{2}}{8 \pi}=\frac{T / 2}{1+k^{2} \lambda_{D}^{2} / S^{2}},
$$

where $\mathbf{E}$ is the electric field, $\lambda_{D}$ is the electron Debye length, $S(\mathbf{k}) \equiv e^{-k^{2} a^{2} / 2}$ is the shape factor associated with the Gaussian particle size $a$ and $V$ is the volume. Therefore, by using particles with the size of a grid in the simulation, the effects on the thermal fluctuation is negligible for the wavelengths of interest, i.e., $k^{2} \lambda_{D}^{2} \ll 1$. Here, the total noise is $T / 2$ and it resides almost entirely in the plasmas waves $\left(\omega= \pm \omega_{p e}\right)$. The use of finite-size particles essentially modifies the Coulomb potential inside the Debye length as shown in Fig. 1. As such, the simulation plasma becomes collisionless and the collisions should then be re-introduced as subgrid phenomena [13]. It is interesting to point out that the introduction of finite-size particles actually fulfills the requirement of $g\left[\equiv 1 / n \lambda_{D}^{3}\right] \ll 1 x s$ without using the actual number density $n$ as in a real plasma. The fluctuation level of the convective cells in 2D magnetized plasmas with the frequency of $\omega \approx i k_{\perp}^{2} \mu_{i}$ has also been numerically verified with [2]

$$
V \frac{|\mathbf{E}(\mathbf{k})|^{2}}{8 \pi}=\frac{T / 2}{1+\omega_{p i}^{2} / \Omega_{i}^{2}+\omega_{p e}^{2} / \Omega_{e}^{2}},
$$




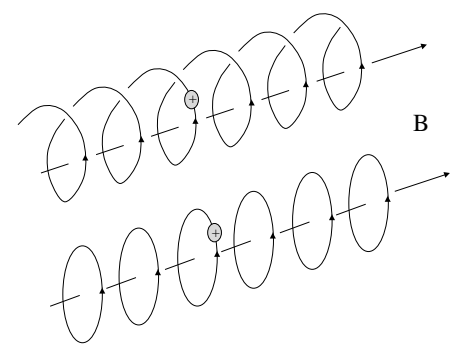

Fig. 2. Gyrokinetic approximation decouples gyrocenter motion from the gyromotion

where $\mu_{i} \equiv 0.3 c_{s}^{2} / \Omega_{i}^{2} \tau_{i}$ is the ion gyroviscosity, $\tau$ is the ion-ion collision time. Here, the fluctuation level is only a small part of the thermal fluctuation with the rest residing in high frequency normal modes such as lower-hybrid waves in this case.

When gyrokinetic particle simulation was first introduced by separating the particle gyromotion from its gyrocenter motion shown in Fig. 2 and by incorporating ion polarization density in Poisson's equation, it was noticed that the noise level went down considerably $[3,4]$. This observation was subsequently verified theoretically [5] as the consequence of a new vacuum result for the dielectric function and the corresponding fluctuation level in an electrostatic gyrokinetic plasmas for $k_{\perp}^{2} \rho_{s}^{2} \ll 1$ is reduced to

$$
V \frac{|\mathbf{E}(\mathbf{k})|^{2}}{8 \pi}=\frac{\lambda_{D}^{2}}{\rho_{s}^{2}}(T / 2),
$$

where $\rho_{s}$ is the ion gyroradius based on the electron temperature, which is much larger than $\lambda_{D}$ in tokamak plasmas. One way to view this property is that the Debye shielding $\left(\lambda_{D}\right)$ as shown in Fig. 1 is now replaced by gyroradius $\left(\rho_{s}\right)$ shielding due to the presence of ion polarization density in the gyrokinetic Poisson's equation. Interestingly, it was found that this level of fluctuation remains the same even for a finite- $\beta$ gyrokinetic plasma $[6,7]$. This unique feature was suspected as the cause for the excessive noise observed in the attempts to simulate shear-Alfvén waves and finite- $\beta$ modified drift waves and ion temperature gradient drift modes using gyrokinetic particle simulation techniques $[6,8]$. Recently, this suspicion was re-visited using the same techniques as those in Ref. [2] and it was found that the fluctuation level associated with shear-Alfvén normal modes is indeed a function of plasma $\beta$ [9], i.e.,

$$
V \frac{|\mathbf{E}(\mathbf{k})|^{2}}{8 \pi}=\frac{\lambda_{D}^{2}}{\rho_{s}^{2}} \frac{T / 2}{1+\omega_{p e}^{2} / c^{2} k^{2}}
$$


for the cold electrons with

$$
\omega= \pm \frac{k_{\|} v_{A}}{\sqrt{1+c^{2} k^{2} / \omega_{p e}^{2}}},
$$

where $c / \omega_{p e}\left[\equiv \rho_{s} \sqrt{m_{e} / m_{i} \beta}\right]$ is the electron skin depth and $\beta \equiv c_{s}^{2} / v_{A}^{2}$ and $v_{A} \equiv c \lambda_{D} / \rho_{s}$ is the Alfvén speed, and

$$
V \frac{|\mathbf{E}(\mathbf{k})|^{2}}{8 \pi}=k^{2} \lambda_{D}^{2} \frac{T / 2}{1+k^{2} \rho_{s}^{2}}
$$

for the warm electrons with

$$
\omega= \pm k_{\|} v_{A} \sqrt{1+k^{2}}
$$

Thus, the fluctuation level reduces as $\beta$ increases in the cold electron limit and is independent of $\beta$ in the warm (adiabatic) electron limit, where the fluctuation level is minimum corresponding to the response of ion acoustic waves. By comparing Eq. (3) with Eqs. (4) and (5), one can see that most of the numerical noise resides outside the normal modes of shear-Alfvén waves of the simulation plasma for large $\beta$. This is a highly unusual situation in the history of particle simulation. Apparently, we have done such a good job in eliminating high frequency waves from the simulation plasma such that the damping mechanism for the fast-moving particles does not exist anymore. This presents a numerical challenge for us. The remedy is to make sure that these fast-moving particles always behavior adiabatically in response to the waves. It can be accomplished through the use of a split-weight particle simulation scheme [9] with

$$
F=F_{0}+\delta f=F_{0}+\frac{e \psi}{T_{e}} F_{0}+\delta h
$$

where $F_{0}$ is the background Maxwellian,

$$
\psi \equiv \phi+(1 / c) \int \partial A_{\|} / \partial t d x_{\|}
$$

$\phi$ is the electrostatic potential and $A_{\|}$is the vector potential parallel to the ambient magnetic field, $\mathbf{B}_{0}$. Simulation results using such a scheme have successfully verified the theoretical predictions of Eqs. (4) and (5). The reduction of noise in the high $\beta$ regime can be viewed as the result of adiabatic electron shielding replacing the ion polarization shielding, which, in turn, replaces Debye shielding as shown in Fig. 1. 
Recently, an investigation on the properties of compressional Alfvén waves [10] in the gyrokinetic formalism has shown that these numerically troublesome high frequency waves can be neglected if the waves of interest have the property of $\omega^{2} / k^{2} v_{A}^{2} \ll 1$. Unlike the difficulties encountered in the MHD simulation, we can do so without any additional geometric ordering. Furthermore, it is found that

$$
\frac{A_{\perp}}{A_{\|}} \sim \frac{\omega^{2}}{k^{2} v_{A}^{2}} \ll 1
$$

where $\mathbf{A}_{\perp}$ is the vector potential perpendicular to $\mathbf{B}_{0}$. Moreover, as indicated in Ref. [10] (see Appendix A), the noise generation by the compressional Alfvén waves is expected to be negligible since $\mathbf{A}_{\perp}$ does not appear in the linear dielectric function. This is quite contrary to the situation for the plasma (space charge) waves of $\omega_{p e}$ in an unmagnetized plasma, for which the associated noise level is much higher than the low-frequency ion acoustic waves, $c_{s}$. As such, implicit schemes had to be used to suppress those waves $[14,15]$.

As we can see, noise properties are of the ultimate concern for the particle simulation. Birdsall and Langdon [16] have paid great attention to this subject in their book and given it a very interesting perspective: "one man's noise is another man's signal." We might add that proper treatment of noise is essential for the success of particle simulation. Hopefully, we have demonstrated here that, as we march into the fully electromagnetic regime, the noise properties of the simulation plasmas are relatively well understood.

\section{Other important properties for microinstability simulations}

However, there are a few outstanding problems. For example, a recent investigations on the ion temperature gradient (ITG) drift turbulence based on gyrokinetic ions and adiabatic electron model have found that energy conservation is not totally satisfactory [17]. First of all, one has to keep the velocity nonlinearity of the ions in order to satisfy

$$
\frac{d}{d t}\left\langle\frac{m_{i}}{2} \int v_{\|}^{2} \delta f_{i} d v_{\|}+\frac{\rho_{s}^{2}}{\lambda_{D}^{2}} \frac{\left|\nabla_{\perp} \phi\right|^{2}}{8 \pi}+\frac{1}{\lambda_{D}^{2}} \frac{\phi^{2}}{8 \pi}\right\rangle=0
$$

where $\langle\cdots\rangle$ is the volume average. It is conceivable that this problem may be again caused by the numerical noise which cannot be damped effectively by the ion Landau damping for the fast-moving ions. In this case, the split-weight scheme for the ions may help [18]. The numerical difficulties encountered in solving Ampere's law in the finite- $\beta$ gyrokinetic particle simulation [19] may 
again trace back to the noise generated by the fast particles. Understanding of these problem are vital if we plan to simulate microinstabilities in the turbulent steady state. The issue of velocity space nonlinearity for both the electron and ions is also very important for nonlinear turbulence simulation. Specifically, it gives rise to velocity space trapping $\left(\omega \approx k_{\| v_{t \alpha}}\right)[20,21]$, which may be important in the presence long-thin eddies when $E \times B$ trapping $\left(\omega \approx 4 k_{\theta} k_{r} \phi\right)[22]$ is not effective.

Another numerical property is the entropy production, which relates the particle or energy flux due to microturbulence to the rate of change of $\delta f^{2}$ through [23]

$$
\frac{1}{2} \frac{\partial}{\partial t} \sum_{\alpha}\left\langle\int\left(\frac{\delta f_{e}^{2}}{F_{e M}}+\frac{\delta f_{i}^{2}}{\tau F_{i M}}\right) d v_{\|}\right\rangle=\frac{1+\tau}{\tau} \kappa_{n}\left\langle\Gamma_{r}\right\rangle,
$$

where $\tau$ is the temperature ratio, $\delta f \equiv F-F_{M}, \Gamma_{r}$ is the radial particle flux, $\kappa_{n}$ is the density inhomogeneity and $\langle\cdots\rangle$ is the flux surface average. Equation (7) reveals two important properties: 1) $\delta f$ would increase indefinitely due to diffusion and, therefore, the $\delta f$ method cannot be used for simulating microturbulence in transport time scale when particle may diffuse more than one density scale length, and 2) the rate of change of the quadratic terms could give us the information on how fast the turbulence is approaching steady-state before we can terminate the simulation. The global gyrokinetic particle simulations on toroidal ITG turbulence have shown that the steady state can be reached relatively fast from the initial quiescent state, say, within 10 - 20 eddy turnover time as observed in the simulations reported in Ref. [24] and those by the global Gyrokinetic Turbulence Code (GTC) in Ref. [25].

So far we have only addressed the issues related to the core transport. However, for simulating core transport barrier and edge turbulence, additional considerations are needed, e.g., the presence of equilibrium $\mathbf{E} \times \mathbf{B}$ flow [26]. Most problematic here is that the resulting gradient scale lengths of the plasma inhomogeneity, $L_{p}$, may become comparable to the Larmor radius, $\rho_{s}$. As such, one needs to take a closer look at the basic ordering used in the original analyses. For example, based on Ref. [3], the gyrokinetic Vlasov equation was derived by (1) changing the phase-space variables from $(\mathbf{x}, \mathbf{v}, t)$ to $\left(\mathbf{R}, v_{\|}, \mu, \varphi, t\right),(2)$ applying gyrokinetic ordering, and (3) then performing gyrophase averaging, where $\mathbf{x}=\mathbf{R}+\rho, \mu \equiv v_{\perp}^{2} / 2 B$ and subscripts $\|$ and $\perp$ denote directions associated with the external $\mathbf{B}$ field. Since the only ordering one needs to separate the gyromotion from the gyrocenter motion is $\rho_{i} / L_{B} \sim o(\epsilon)$ [27], we can show that, by following the procedures in Ref. [3], the only conditions we need to recover the original gyrokinetic Vlasov equation are:

$$
\frac{\partial F}{\partial \varphi}=\frac{\partial F}{\partial \mu}=0
$$




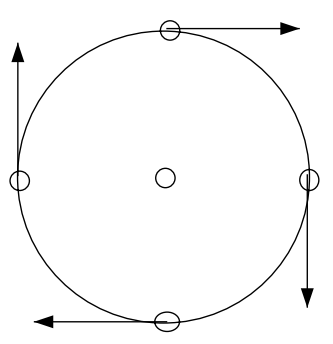

Fig. 3. Four-point approximation for a rotating ion ring

Thus, as long as we are interested in low frequency phenomena and the gyrophase information is unimportant and the magnetic moments are conserved, we can still use low-frequency gyrokinetic particle simulation for investigating the physics in these regimes. As far as collisions are concerned, the GTC code already has the capability to handle e-i, e-e and i-i collisions [28] and has been used for neoclassical transport studies [29]. Thus, in principle, global gyrokinetic particle codes can be used for full cross section simulations including both the core and the edge. Of course, many details have to be worked out for the present GTC code for such an extension.

Moreover, the recent work [10] exploring the relationship between MHD and gyrokinetics has shown that the gyrokinetic formalism enables us to keep the FLR effects in the gyrocenter coordinates by representing a gyrokinetic particle as a rotating ring in the kinetic-MHD regime. Shown in Fig. 3 is the 4-point approximation of the ring valid for $k_{\perp} \rho_{i} \leq 2$. In other words, we have found a way to keep FLR effects in the MHD regime when the grid spacing of $\rho_{s}$ becomes necessary. These findings are very encouraging in terms of expanding the applicability of the gyrokinetic particle simulation to the regime traditionally studied by the MHD equations. One example is the possibility of microturbulence simulation in transport time scale. Let us explain.

\section{Transport scale simulation of microturbulence}

In a recent study on size scaling [30] associated with the ITG turbulence, the simulation was carried out for a reactor size plasma with $a / \rho_{s}=1000$ on the IBM-SP at National Energy Research Supercomputing Center (NERSC). The run took about 72 wallclock hours with one billion ion particles ( 8 particles / cell) for 7,000 time steps running on 1024 processors (25M particles*step/sec) with $10 \%$ efficiency for each processor. This is impressive. However, the simulation covers only $1 \mathrm{msec}$ of the discharge in real time. Thus, the confinement time scale simulation of $\sim 1 \mathrm{sec}$ is almost impossible with the present-day computing capability. There are other numerical difficulties preventing us from doing so as well as we mentioned earlier. So, the scenario of transport time scale simulation can go like this: 
1) Load the particles according to the given magnetic equilibrium and plasma profiles of density, parallel current and pressure and run the microturbulence simulation as an initial value problem.

2) Calculate the flux surface-averaged transport coefficients for density, current and temperature from the simulation and then use, e.g., the TRANSP code [31] to predict the new profiles, for the next $50-100$ msec.

3) Calculate the new magnetic equilibrium using codes such as JSLOVER [32] or PIES [33].

4) Re-load particles accordingly and go to step 1.

The governing equations related to the proposed simulation model is given in Appendix A.

\section{Conclusions}

For the past ten years, particle simulation has proven to be very adaptable to massively parallel computers, for both those with commodity processors and the vector-parallel machines [6,34]. The major reason is that particle simulation solves a set of linear ordinary differential equations in the Lagrangian coordinates and only solve the elliptic-type partial differential equation in the Eulerian coordinates. As such, particle simulation keeps inter-processor communication at a minimum and spends most of time performing gather-scatter operations. A special built machine for particle pushing designed with these special features in mind to simulate International Tokamak Experimental Reactor (ITER) physics is an intriguing option. Success of such an endeavor is a fitting tribute to the pioneers of particle simulation.

\section{Acknowledgment}

This work supported by Contract No. DE-AC02-CHO-3073 and and the SciDAC Plasma Turbulence Project of the US DoE. The author would like to thank Dr. T. S. Hahm, Dr. S. Hudson, Dr. D. Monticello, Dr. M. Redi, and Dr. G. Rewoldt for useful discussions.

\section{A Governing equations for gyrokinetic particle simulation}

The governing equations for the microturbulence and kinetic-MHD simulation are similar to those described in Ref. [10]. The gyrokinetic Vlasov equation in 
the gyrocenter coordinates of $\left(\mathbf{R}, v_{\|}, \mu, t\right)$ can be written as

$$
\frac{\partial F_{\alpha g c}}{\partial t}+\frac{d \mathbf{R}}{d t} \cdot \frac{\partial F_{\alpha g c}}{\partial \mathbf{R}}+\frac{d v_{\|}}{d t} \frac{\partial F_{\alpha g c}}{\partial v_{\|}}=0,
$$

where $\mathbf{R}=\mathbf{x}-\rho$. In general geometry including electromagnetic and toroidal effects for finite $k_{\perp} \rho_{\alpha}$, the corresponding characteristics are

$$
\begin{aligned}
& \frac{d \mathbf{R}}{d t}=v_{\|} \mathbf{b}^{*}+\frac{v_{\perp}^{2}}{2 \Omega_{\alpha 0}} \hat{\mathbf{b}}_{0} \times \nabla \ln B_{0}-\frac{c}{B_{0}} \nabla \bar{\phi} \times \hat{\mathbf{b}}_{0}, \\
& \frac{d v_{\|}}{d t}=-\frac{v_{\perp}^{2}}{2} \mathbf{b}^{*} \cdot \nabla \ln B_{0}-\frac{q_{\alpha}}{m_{\alpha}}\left(\mathbf{b}^{*} \cdot \nabla \bar{\phi}+\frac{1}{c} \frac{\partial \bar{A}_{\|}}{\partial t}\right), \\
& \mu \equiv \frac{v_{\perp}^{2}}{2 B_{0}}\left(1-\frac{m c}{e} \frac{v_{\|}}{B_{0}} \hat{\mathbf{b}}_{0} \cdot \nabla \times \hat{\mathbf{b}}_{0}\right) \approx \text { cons. }
\end{aligned}
$$

where

$$
\begin{aligned}
& \mathbf{b}^{*} \equiv \mathbf{b}+\frac{v_{\|}}{\Omega_{\alpha 0}} \hat{\mathbf{b}}_{0} \times\left(\hat{\mathbf{b}}_{0} \cdot \nabla\right) \hat{\mathbf{b}}_{0}, \\
& \mathbf{b}=\hat{\mathbf{b}}_{0}+\frac{\nabla \times \overline{\mathbf{A}}}{B_{0}}, \\
& F_{\alpha g c}=\sum_{j=1}^{N_{\alpha}} \delta\left(\mathbf{R}-\mathbf{R}_{\alpha j}\right) \delta\left(\mu-\mu_{\alpha j}\right) \delta\left(v_{\|}-v_{\| \alpha j}\right),
\end{aligned}
$$

$N_{\alpha}$ is the total number of particles, $\rho_{\alpha}$ is the gyroradius of the species $\alpha$, $\Omega_{\alpha 0} \equiv q_{\alpha} B_{0} / m_{\alpha} c$ and the gyrophase averaged potentials are

$$
\left(\begin{array}{c}
\bar{\phi} \\
\overline{\mathbf{A}}
\end{array}\right)(\mathbf{R})=\left\langle\int\left(\begin{array}{l}
\phi \\
\mathbf{A}
\end{array}\right)(\mathbf{x}) \delta(\mathbf{x}-\mathbf{R}-\rho) d \mathbf{x}\right\rangle_{\varphi}
$$

with $\langle\cdots\rangle_{\varphi} \equiv \oint d \varphi / 2 \pi$. Gyrokinetic Poisson's equation can be written as

$$
\left(\tau / \lambda_{D}^{2}\right)[\phi(\mathbf{x})-\tilde{\phi}(\mathbf{x})]=-4 \pi \rho_{g c}
$$

where

$$
\begin{aligned}
& \tilde{\phi}(\mathbf{x}) \equiv\left\langle\int \bar{\phi}(\mathbf{R}) F_{i}\left(\mathbf{R}, \mu, v_{\|}\right) \delta(\mathbf{R}-\mathbf{x}+\rho) d \mathbf{R} d \mu d v_{\|}\right\rangle_{\varphi} \\
& \rho_{g c}(\mathbf{x})=\sum_{\alpha} q_{\alpha}\left\langle\int F_{\alpha g c}(\mathbf{R}) \delta(\mathbf{R}-\mathbf{x}+\rho) d \mathbf{R} d v_{\|} d \mu\right\rangle_{\varphi}
\end{aligned}
$$


$\tau \equiv T_{e} / T_{i}, \lambda_{D} \equiv \sqrt{T_{e} / 4 \pi n_{0} e^{2}}$ and $\bar{\phi}$. Gyrokinetic Ampere's law becomes

$$
\nabla^{2} \mathbf{A}-\frac{1}{v_{A}^{2}} \frac{\partial^{2} \mathbf{A}_{\perp}}{\partial t^{2}}=-\frac{4 \pi}{c} \mathbf{J}_{g c}
$$

where

$$
\begin{aligned}
& \mathbf{J}_{g c}(\mathbf{x})=\mathbf{J}_{\| g c}(\mathbf{x})+\mathbf{J}_{\perp g c}^{M}(\mathbf{x})+\mathbf{J}_{\perp g c}^{d}(\mathbf{x}), \\
& =\sum_{\alpha} q_{\alpha}\left\langle\int\left(\mathbf{v}_{\|}+\mathbf{v}_{\perp}+\mathbf{v}_{d}\right) F_{\alpha g c}(\mathbf{R}) \delta(\mathbf{R}-\mathbf{x}+\rho) d \mathbf{R} d v_{\|} d \mu\right\rangle_{\varphi},
\end{aligned}
$$

$\mathbf{v}_{\|}$and $\mathbf{v}_{\perp}$ are the velocity space variables and

$$
\mathbf{v}_{d} \equiv \frac{v_{\|}^{2}}{\Omega_{\alpha 0}} \hat{\mathbf{b}}_{0} \times\left(\hat{\mathbf{b}}_{0} \cdot \nabla\right) \hat{\mathbf{b}}_{0}+\frac{v_{\perp}^{2}}{2 \Omega_{\alpha 0}} \hat{\mathbf{b}}_{0} \times \nabla \ln B_{0} .
$$

For $\omega^{2} \ll k^{2} v_{A}^{2}$, we can simply ignore the time derivative term in gyrokinetic Ampere's law without having to invoke any further geometric simplifications.

The gyrophase averages in Eqs. (A.1) - (A.4) can be calculated by the scheme described in Fig. 1, namely,

$$
\begin{aligned}
& \left(\begin{array}{c}
\bar{\phi} \\
\overline{\mathbf{A}}
\end{array}\right)\left(\mathbf{R}_{\alpha j}\right)=\left\langle\left(\begin{array}{c}
\phi \\
\mathbf{A}
\end{array}\right)\left(\mathbf{x}_{\alpha j}\right)\right\rangle_{\varphi}, \\
& \rho_{g c}(\mathbf{x})=\sum_{\alpha} q_{\alpha} \sum_{j=1}^{N}\left\langle\delta\left(\mathbf{x}-\mathbf{x}_{\alpha j}\right)\right\rangle_{\varphi}, \\
& \mathbf{J}_{\| g c}(\mathbf{x})=\sum_{\alpha} q_{\alpha} \sum_{j=1}^{N} v_{\| \alpha j}\left\langle\delta\left(\mathbf{x}-\mathbf{x}_{\alpha j}\right)\right\rangle_{\varphi}, \\
& \mathbf{J}_{\perp g c}^{d}(\mathbf{x})=\sum_{\alpha} q_{\alpha} \sum_{j=1}^{N} \mathbf{v}_{d \alpha j}\left\langle\delta\left(\mathbf{x}-\mathbf{x}_{\alpha j}\right)\right\rangle_{\varphi}
\end{aligned}
$$

and

$$
\mathbf{J}_{\perp g c}^{M}(\mathbf{x})=\sum_{\alpha} q_{\alpha} \sum_{j=1}^{N}\left\langle\mathbf{v}_{\perp \alpha j} \delta\left(\mathbf{x}-\mathbf{x}_{\alpha j}\right)\right\rangle_{\varphi}
$$

The significance here is that the FLR effects can now be accounted for by using the scheme for all the field quantities including the perpendicular current. 
Since we assume that $\rho / L_{e q}$ is of the order $o(\epsilon)$, the magnetic drift current, in the limit of small $k_{\perp} \rho_{i}$, can be calculated explicitly as

$$
\mathbf{J}_{\perp g c}^{d}=\frac{c}{B_{0}} \sum_{\alpha}\left[p_{\alpha \|}\left(\nabla \times \hat{\mathbf{b}}_{0}\right)_{\perp}+p_{\alpha \perp} \hat{\mathbf{b}}_{0} \times\left(\nabla \ln B_{0}\right)\right],
$$

and the diamagnetic current as

$$
\mathbf{J}_{\perp g c}^{M}=-\sum_{\alpha} \nabla \times \frac{c \hat{\mathbf{b}}_{0}}{B_{0}} p_{\alpha \perp},
$$

where

$$
\left(\begin{array}{c}
p_{\alpha \|} \\
p_{\alpha \perp}
\end{array}\right)=m_{\alpha} \int\left(\begin{array}{c}
v_{\|}^{2} \\
v_{\perp}^{2} / 2
\end{array}\right) F_{\alpha g c}(\mathbf{x}) d v_{\|} d \mu .
$$

For $p=p_{\alpha \|}=p_{\alpha \perp}$, we recover the usual pressure balance equation as

$$
\mathbf{J}_{\perp g c}=\frac{c}{B_{0}} \sum_{\alpha} \hat{\mathbf{b}}_{0} \times \nabla p_{\alpha} .
$$

These equations can be solved via existing particle simulation techniques on massively parallel computers.

\section{References}

[1] A. B. Langdon and C. K. Birdsall, Phys. Fluids 13, 2115 (1970).

[2] H. Okuda and J. M. Dawson, Phys. Fluids 16, 408 (1973).

[3] W. W. Lee, Phys. Fluids 26, 556 (1983).

[4] W. W. Lee, J. Comp. Phys. 72, 243 (1987).

[5] J. A. Krommes, W. W. Lee and C. Oberman, Phys. Fluids 29 2421(1986).

[6] J. V. W. Reynders, Ph.D. thesis, Princeton University (1992).

[7] J. A. Krommes, Phys. Rev. Lett. 70, 3067(1993); Phys. Fluids B 5, 2404 (1993); 5, 1066 (1993).

[8] J. .C. Cummings, Ph.D. thesis, Princeton University (1995).

[9] W. W. Lee, J. L. V. Lewandowski, T. S. Hahm, and Z. Lin, Phys. Plasmas 8, 4435 (2001). 
[10] W. W. Lee and H. Qin, Phys. Plasmas, (2003).

[11] J. M. Dawson, C. G. Hsi, and R. Shanny, Bull. Am. Phys. Soc. 13, 1744 (1968).

[12] C. K. Birdsall, A. N. Langdon, C. F. McKee, H. Okuda, and D. Wong, Bull. Am. Phys. Soc. 13, 174 (1968).

[13] R. Shanny, J. M. Dawson and J. M. Greene, Phys. Fluids 101281 (1967).

[14] A. Friedman, A. B. Langdon, and B. I. Cohen, Comments Plasma Phys. Controlled Fusion, 6, 225 (1981).

[15] J. Denavit, J. Comp. Phys. 42, 337 (1981).

[16] C. K. Birdsall and A. B. langdon, Plasma Physics via Computer Simulation, Adam Hilger, New York (1991).

[17] R. Hatzky, T. M. Tran, A. Konies, R. Kleiber, S. J. Allfrey, Phys. Plasmas 9 898 (2002).

[18] I. Manuilskiy and W. W. Lee, Phys. Plasmas 7, 1381 (2000).

[19] Y. Chen and S. E. Parker, J. Comput. Phys. (to be published).

[20] T. M. O’Neil, Phys. Fluids 8, 2255 (1965)

[21] S. E. Parker and W. W. Lee, Phys. Fluids B 5, 77 (1993).

[22] A. M. Dimits and W. W. Lee, Phys. Fluids B 3, 1557 (1991).

[23] W. W. Lee and W. M. Tang, Phys. Fluids 31, 612 (1988).

[24] S. E. Parker, W. W. Lee, and R. A. Santoro Phys. Rev. Lett. 71, 2042 (1993)

[25] Z. Lin, T. S. Hahm, W. W. Lee, W. M. Tang, and R. B. White, Science 281, 1835 (1998).

[26] T. S. Hahm, Phys. Plasmas 3, 4658 (1996).

[27] H. Qin, W. M. Tang, W. W. Lee, Phys. Plasmas 7, 4433 (2000).

[28] Z. Lin, T. S. Hahm, W. W. Lee, W. M. Tang, P. H. Diamond, Phys. Rev. Lett. 83, 3645 (1999).

[29] Z. Lin, W. M. Tang, W. W. Lee, Phys. Plasmas 2, 2975 (1995).

[30] Z. Lin, S. Ethier, T. S. Hahm, and W. M. Tang, Phys. Rev. Lett. 88, 195004 (2002).

[31] http://w3.pppl.gov/transp/

[32] J. DeLucia, S. C. Jardin, and A. M. M. Todd, J. Comput. Phys. 372 (1980).

[33] A. Reiman and H. Greenside, Comp. Phys. Comm. 43, 157 (1986).

[34] S. Ethier, this conference. 


\section{External Distribution}

Plasma Research Laboratory, Australian National University, Australia

Professor I.R. Jones, Flinders University, Australia

Professor João Canalle, Instituto de Fisica DEQ/IF - UERJ, Brazil

Mr. Gerson O. Ludwig, Instituto Nacional de Pesquisas, Brazil

Dr. P.H. Sakanaka, Instituto Fisica, Brazil

The Librarian, Culham Laboratory, England

Mrs. S.A. Hutchinson, JET Library, England

Professor M.N. Bussac, Ecole Polytechnique, France

Librarian, Max-Planck-Institut für Plasmaphysik, Germany

Jolan Moldvai, Reports Library, Hungarian Academy of Sciences, Central Research Institute for Physics, Hungary

Dr. P. Kaw, Institute for Plasma Research, India

Ms. P.J. Pathak, Librarian, Institute for Plasma Research, India

Ms. Clelia De Palo, Associazione EURATOM-ENEA, Italy

Dr. G. Grosso, Instituto di Fisica del Plasma, Italy

Librarian, Naka Fusion Research Establishment, JAERI, Japan

Library, Laboratory for Complex Energy Processes, Institute for Advanced Study, Kyoto University, Japan

Research Information Center, National Institute for Fusion Science, Japan

Dr. O. Mitarai, Kyushu Tokai University, Japan

Dr. Jiangang Li, Institute of Plasma Physics, Chinese Academy of Sciences, People's Republic of China

Professor Yuping Huo, School of Physical Science and Technology, People's Republic of China

Library, Academia Sinica, Institute of Plasma Physics, People's Republic of China

Librarian, Institute of Physics, Chinese Academy of Sciences, People's Republic of China

Dr. S. Mirnov, TRINITI, Troitsk, Russian Federation, Russia

Dr. V.S. Strelkov, Kurchatov Institute, Russian Federation, Russia

Professor Peter Lukac, Katedra Fyziky Plazmy MFF UK, Mlynska dolina F-2, Komenskeho Univerzita, SK-842 15 Bratislava, Slovakia

Dr. G.S. Lee, Korea Basic Science Institute, South Korea

Institute for Plasma Research, University of Maryland, USA

Librarian, Fusion Energy Division, Oak Ridge National Laboratory, USA

Librarian, Institute of Fusion Studies, University of Texas, USA

Librarian, Magnetic Fusion Program, Lawrence Livermore National Laboratory, USA

Library, General Atomics, USA

Plasma Physics Group, Fusion Energy Research Program, University of California at San Diego, USA

Plasma Physics Library, Columbia University, USA

Alkesh Punjabi, Center for Fusion Research and Training, Hampton University, USA

Dr. W.M. Stacey, Fusion Research Center, Georgia Institute of Technology, USA

Dr. John Willis, U.S. Department of Energy, Office of Fusion Energy Sciences, USA

Mr. Paul H. Wright, Indianapolis, Indiana, USA 
The Princeton Plasma Physics Laboratory is operated by Princeton University under contract with the U.S. Department of Energy.

\author{
Information Services \\ Princeton Plasma Physics Laboratory \\ P.O. Box 451 \\ Princeton, NJ 08543
}

Phone: 609-243-2750

Fax: 609-243-2751

e-mail: pppl_info@pppl.gov

Internet Address: http://www.pppl.gov 